\title{
Worst case analysis of random circuits using Taylor models and Bernstein polynomials techniques
}

\author{
Yuchao Guo, Jingyi Cao, Xiaoxu Wang, Tongyu Ding*, Liang Zhang \\ College of Information Engineering, Jimei University \\ 401 Keli Building, 183 Yinjiang Road \\ Xiamen, China \\ tyding@jmu.edu.cn
}

\begin{abstract}
In this paper, a novel paradigm to optimize the Taylor models based worst-case analysis results of random circuits responses in time domain has been proposed. The methodology leverages the so-called Bernstein polynomials to yield a conservative, yet tight, prediction of the worst case bounds. This proposed framework has been used and verified in the time-domain analysis of an exemplary linear circuit, which demonstrated its feasibility and strength.
\end{abstract}

Keywords-worst case analysis; Taylor models; Bernstein polynomials; reliability; uncertainty

\section{Introduction}

Circuits and system variability has long been impacting the stability and reliability of electrical and electronic equipment, and only in the last century or so have scientists and engineers been able to investigate the responses of electronic devices affected by unknown uncertainties via numerical techniques [1] This pursuit was made possible through advancements in computer techniques [2-3]. Among these numerical methods, Taylor models (TM), as a sophisticated approach combining the force of the traditional interval analysis (IA) method, offers reasonable inherent worst case (WC) responses of circuits and systems in a timely fashion [4-6]. In the TM operations, all random parameters are represented in terms of truncated Taylor expansions and an interval variable, while the interval variable accounts for the truncation errors. The corresponding TM operations abide both polynomial and IA rules. The final range of the response is provided by both the polynomial and the IAremainder, hence it is indispensable to determine the exact bound of this truncated polynomial, which is indeed non-trivial, especially for high-order multivariate polynomials [6]. A rough approximation of the polynomial bound will possibly lead to unnecessarily strict margins, which are very unlikely to occur [3].

In order to improve the TM based simulation results, one idea is to adopt more sophisticated bound functions for the polynomial part. Bernstein polynomials play an extremal position in some classes of operations [7]. It is capable of determining somehow tight bounds of a multivariate polynomial over a closed box, and is therefore widely used for global optimization [8] and numerical approximation [9]. The aim of this paper is to outline how Bernstein basis helps improve the TM-based WC analysis of passive linear circuits in time domain.

\section{Taylor Models}

The idea of the so called TM approach is to represent a nonlinear function as Taylor polynomials combining an interval remainder. For instance, given $f$ of an interval variable $\bar{x}=[a, b]$ (with the center of $\bar{x}$ being $\left.x_{0}=(a+b) / 2\right)$, The TM form of $f(\bar{x})$ around $x_{0}$ should be

$$
f(\bar{x})=P_{f}\left(x-x_{0}\right)+I_{f}
$$

where

$$
P_{f}\left(x-x_{0}\right)=\sum_{k=0}^{n} \frac{f^{(k)}\left(x_{0}\right)}{k !}\left(x-x_{0}\right)^{k}
$$

is the expanded polynomial part of $f(x)$ around $x_{0}$, and $I_{f}$ being an interval value, which is commonly defined as the Lagrange remainder of (2), the readers are suggested to see [45] for detailed discussion on the calculation of IA-remainder.

Denote $B(\cdot)$ as the operator to calculate the bound of a function, and the overall bound of the TM form function can be represented as

$$
B(f(\bar{x}))=B\left(P_{f}\right)+I_{f}
$$

This overall bound is obtained from the IA-sum of two subintervals, and it should enclose $f(x)$ between two curves for $\forall x \in[a, b]$. Ideally, $I_{f}=[0,0]$ implies that $P_{f}(x)$ provides an exact parametric representation of $f(x)$, however in reality, the TM form bound of (3) always provides over-estimated results.

Given two TM form functions $f(\bar{x})=P_{f}(x)+I_{f}$ and $g(\bar{x})=P_{g}(x)+I_{g}$, we can readily write the algebraic operations between them, for instance, the product between them yields

$$
\begin{aligned}
u(\bar{x}) & =f(\bar{x}) g(\bar{x})=\left(P_{f}(x)+I_{f}\right)\left(P_{g}(x)+I_{g}\right) \\
& =P_{f}(x) P_{g}(x)+P_{f}(x) I_{g}+P_{g}(x) I_{f}+I_{f} I_{g}
\end{aligned}
$$


The first term in (4) can be further expressed as

$$
P_{f}(x) P_{g}(x)=P_{u}(x)+P_{e}(x)
$$

where the order of $P_{u}(x)$ is equal to that of $P_{f}(x)$ and $P_{g}(x)$, thus accounting for the polynomial part of the new TM form of $u(x)$, whilst $P_{e}(x)$ accounts for the higher-order contribution. In this sense, the new remainder of the product includes four remaining terms and should be calculated as follows.

$$
I_{u}=B\left(P_{e}\right)+B\left(P_{f}\right) I_{g}+B\left(P_{g}\right) I_{f}+I_{f} I_{g}
$$

It is worth noting that the use of Taylor models requires a fast and accurate bound operator $B(\cdot)$ of the polynomials. Since IA has been included in each step of the TM operations, a rough bound of the polynomial part may lead to a large overestimation in a long chain of realistic operations. In the univariate case, the bounds of a polynomial can be readily obtained with analytical approaches, while in the multivariate case it is non-trivial and requires suitable solutions. One possible solution is introduced in the next part.

\section{Multivariate Polynomial Bounds}

As has been stated in the previous section, at the end of a series of TM operations, we have obtained a TM form function, the polynomial part of which is commonly represented by the so called power basis

$$
P(x)=\sum_{i=0}^{l} a_{i} x^{i}, \quad x^{i}=\prod_{\mu=1}^{n} x_{\mu}^{i_{\mu}}
$$

in $x_{1}, \ldots, x_{n}$, of the degree $l=\left(l_{1}, \ldots, l_{n}\right)$, as well as a box

$$
X=\left[\underline{x_{1}}, \bar{x}_{1}\right] \times \cdots \times\left[\underline{x_{n}}, \bar{x}_{n}\right]
$$

This is only one of an infinite number of bases for the space of polynomials. In order to obtain a tight outer bound for $P(x)$ over $X$, one method is to convert the power basis form multivariate polynomial (7) into the Bernstein form, which reads:

$$
P(x)=\sum_{i=0}^{l} b_{i} B_{i}(x)
$$

where

$$
B_{i}(x)=\left(\begin{array}{l}
l \\
i
\end{array}\right) x^{i}(1-x)^{l-i}
$$

form a basis, which constitutes the vector space of polynomials with degree $l$, and

$$
b_{i}=\sum_{j=0}^{i} \frac{\left(\begin{array}{c}
i \\
j
\end{array}\right)}{\left(\begin{array}{l}
l \\
j
\end{array}\right)} a_{j}, \quad 0 \leq i \leq l
$$

are the so-called Bernstein coefficients. It is relevant to point out that the evaluation of $b_{i}$ in (11) is effective only for unit box, namely $X=[0,1]^{n}$. For any arbitrary non-empty box, the general form of Bernstein coefficients can be found in (12)

$$
b_{i}=\sum_{j=0}^{i} \frac{\left(\begin{array}{c}
i \\
j
\end{array}\right)}{\left(\begin{array}{l}
l \\
j
\end{array}\right)}(\bar{x}-\underline{x})^{j} \sum_{k=j}^{l}\left(\begin{array}{l}
k \\
j
\end{array}\right) \underline{x}^{k-j} a_{k}
$$

Once we obtain the coefficients set $b_{i}$ of the Bernstein expansion for a given polynomial, the range of the polynomial over a particular box is determined simultaneously, which is tightly bounded by these coefficients, as the property states: the range of a polynomial $P$ over a box $X$ is contained between the minimum value and the maximum value of the Bernstein coefficients [6]

$$
\min _{i}\left\{b_{i}\right\} \leq P(x) \leq \max _{i}\left\{b_{i}\right\}, \quad x \in X
$$

Obviously, one has to compute all of the Bernstein coefficients to determine the minimum and maximum, and this can be extremely time-consuming in high-order operations consisting of a relatively large number of variables. Smith proposed a new approach to calculate the B-coefficients of multivariate polynomials in [6], where he begins with multivariate monomials. Consider the following polynomial with only one term

$$
q(x)=a_{k} x^{k}, \quad x=\left(x_{1}, \ldots, x_{n}\right), 0 \leq k \leq l
$$

The Bernstein coefficients for monomial can be modified as

$$
b_{i}=a_{k} \sum_{j=0}^{\min \{i, k\}} \frac{\left(\begin{array}{l}
i \\
j
\end{array}\right)}{\left(\begin{array}{l}
l \\
j
\end{array}\right)}(\bar{x}-\underline{x})^{j}\left(\begin{array}{l}
k \\
j
\end{array}\right) \underline{x}^{k-j}
$$

On condition that a polynomial $P$ is composed of $m$ terms, the overall Bernstein coefficients can be acquired from the sum of all the coefficients of every single monomial. In this case, the computational complexity is simply linear to the number of total polynomials.

\section{Numerical Results}

In this section, we have collected the application results of the proposed technique to the time domain simulation of the passive circuits depicted in Fig. 1, where all component values $R, L$, and $C$ are defined by an interval value with given bounds.

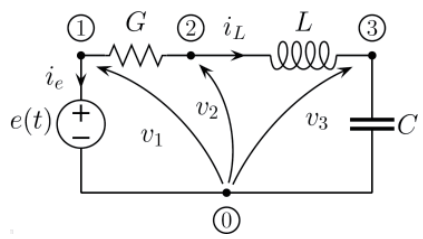

Fig. 1. An exemplary example of RLC circuit used to validate the proposed TM method and Bernstein technique for worst-case analysis of random circuits in time domain. 
The nominal value of the parameters in Fig. 1 is $R_{0}=1 \Omega$, $L_{0}=1 \mathrm{H}$, and $C_{0}=1 \mathrm{~F}$. with all variables a $\pm 10 \%$ tolerance. The circuit equation in form of matrix writes as follows:

$$
\mathbf{Y}_{\mathbf{d}} \frac{d}{d t} \mathbf{w}(t)+\mathbf{Y}_{\mathbf{g}} \mathbf{w}(t)=\mathbf{J}
$$

where $\mathbf{w}=\left[v_{1}(t), v_{2}(t), v_{3}(t), i_{L}(t), i_{e}(t)\right]^{T}$ is the vector of unknown variables, $\mathbf{J}=[0,0,0,0, e(t)]^{T}$, and

$$
\mathbf{Y}_{\mathbf{g}}=\left[\begin{array}{ccccc}
G & -G & 0 & 0 & 1 \\
-G & G & 0 & 1 & 0 \\
0 & 0 & 0 & -1 & 0 \\
1 & 0 & 0 & 0 & 0 \\
0 & 1 & -1 & 0 & 0
\end{array}\right], \quad \mathbf{Y}_{\mathbf{d}}=\left[\begin{array}{ccccc}
0 & 0 & 0 & 0 & 0 \\
0 & 0 & 0 & 0 & 0 \\
0 & 0 & C & 0 & 0 \\
0 & 0 & 0 & 0 & 0 \\
0 & 0 & 0 & 0 & -L
\end{array}\right]
$$

are the interval valued parameter matrices. To solve the differential equation (16), we resort to the custom Trapezoidal rule, namely, if $\frac{d w}{d t}=f(t, w)$, then

$$
\frac{w_{n+1}-w_{n}}{h}=\frac{1}{2}\left[f\left(t_{n}, w_{n}\right)+f\left(t_{n}, w_{n+1}\right)\right]
$$

where $h=t_{n+1}-t_{n}$ is the time step. The final time-domain analysis using sampled-data iterative map is performed as

$$
\left(2 \mathbf{Y}_{\mathrm{d}}+h \mathbf{Y}_{\mathrm{g}}\right) \mathbf{w}_{n+1}=\left(2 \mathbf{Y}_{\mathrm{d}}-h \mathbf{Y}_{\mathrm{g}}\right) \mathbf{w}_{n}+h\left(\mathbf{a}_{n+1}+\mathbf{a}_{n}\right)
$$

Unfortunately, a divergence is observed for the timedomain responses, as can be seen in Fig. 2, due to the unlimited growth of the IA-remainder.

If and only if, we use a higher-order Bernstein bound method, the over-estimation is beat successfully, and the optimized responses of capacitance voltage for the Fig. 1 circuit can be observed as shown in Fig. 3 .

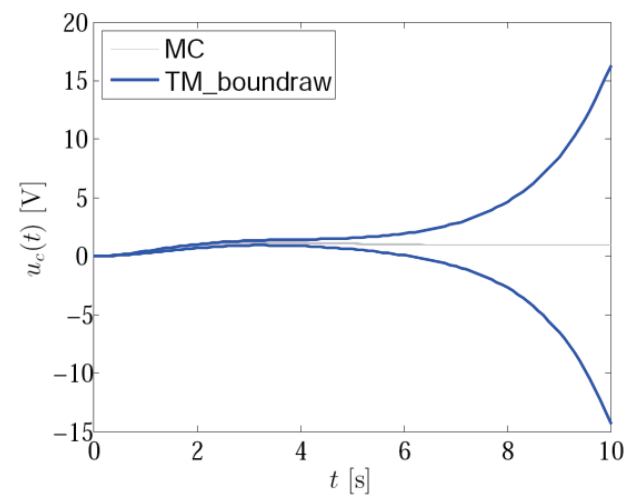

Fig. 2. Error explosion due to the divergence of IA-remainder

\section{Conclusion}

In this work, we have illustrated a Taylor models algorithm framework combing the strength of Bernstein polynomials to jointly obtain the time-domain responses of a random circuit with bounded uncertain parameters. Simulated results demonstrated both the accuracy and the feasibility of the technique, and highlighted that the proposed framework allows for achieving a substantial improvement compared with plain TM calculations.

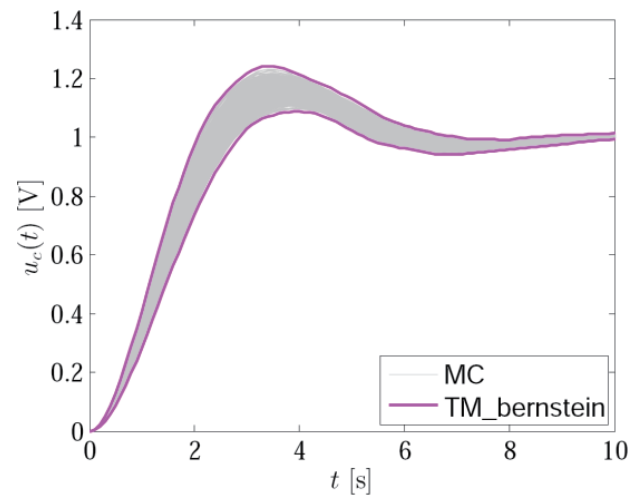

Fig. 3. Time-domain solution of the capacitor voltage. The upper bound and lower bound are obtained by TMs combining Bernstein bound solution (magenta lines), and are compared with 10,000 Monte Carlo samples (gray area).

\section{Acknowledgment}

The authors would like to appreciate the support from Fujian Natural Science Foundation (2017J01505, 2018J05109) and Educational Department of Fujian, China (under grant no. JZ160449).

\section{References}

[1] James Ma and Rob Rutenbar, "Fast interval-valued statistical modeling of interconnect and effective capacitance," IEEE Transactions on Computer-Aided Design of Integrated Circuits and Systems, vol. 25, no. 4, pp. 710-724, Apr. 2006.

[2] Qingyu Yang and Yong Chen, "Monte Carlo methods for reliability evaluation of linear sensor systems", IEEE Transactions on Reliability., vol. 60, no. 1, pp. 305-314, Mar. 2011.

[3] Tongyu Ding, Riccardo Trinchero, Paolo Manfredi, Igor Stievano, and Flavio Canavero, "How affine arithmetic helps beat uncertainties in electrical systems", IEEE Circuits \& Systems Magazine, vol. 15, no. 4, pp. 70-79, Nov. 2015.

[4] Martin Berz and Georg Hoffstatter, "Computation and application of Taylor polynomials with interval remainder bounds," Reliable Computing, vol. 4, no. 1, pp. 83-97, 1998.

[5] K. Makino and M. Berz, "Taylor models and other validated functional inclusion methods," International Journal of Pure \& Applied Mathematics, vol. 4, no. 4, pp. 379-456, 2003.

[6] Riccardo Trinchero, Paolo Manfredi, Tongyu Ding and Igor Stievano, "Combined Parametric and Worst Case Circuit Analysis via Taylor Models," IEEE Transactions on Circuits \& Systems I, Regular Papers, vol. 63, no. 7, pp. 1067-1078, 2016.

[7] Andrew Smith, "Fast construction of constant bound functions for sparse polynomials," Journal of Global Optimization, vol. 43, 2009.

[8] J. Garloff, "Convergent bounds for the range of multivariate polynomials," In: Nickel K. (eds) Interval Mathematics 1985. Lecture Notes in Computer Science, vol 212. Springer, Berlin, Heidelberg.

[9] G. G. Lorentz, Bernstein Polynomials, New York: Chelsea Publishing Company, 1986. 
Recent Developments on Information and

Communication Technology (ICT) Engineering- Meen, Yang \& Zhao ISBN: 978-981-14-2136-5 